\title{
Interaction between chloride ions mediated by carbon nanotubes: a chemical attraction
}

\author{
Fabiola Dominguez-Flores $^{1}$ - Elizabeth Santos ${ }^{1}$ • Wolfgang Schmickler ${ }^{1}$ (D) $\cdot$ Fernanda Juarez $^{1}$
}

Received: 11 July 2020 / Revised: 6 August 2020 / Accepted: 7 August 2020 / Published online: 15 September 2020

(C) The Author(s) 2020

\begin{abstract}
The interaction between two $\mathrm{Cl}^{-}$ions separated by the wall of a narrow carbon nanotube has been investigated by density functional theory (DFT) and by DFT-based tight binding (DFTB+). The direct Coulomb interaction between the ions is screened by the nanotube, no matter if the latter is conducting or semiconducting. The presence of the ions induces changes in the electronic density of states of the nanotube, which results in an effective attraction between the ions of the order of $0.2-0.3 \mathrm{eV}$. The interaction of the outside ions with the tube has a covalent component, when the two ions are near there is even a direct chemical attraction between the ions. In contrast to the effective attraction between two $\mathrm{Li}^{+}$ions reported before (Juarez et al., Phys Chem Chem Phys 22:10,603, 2020), the effect cannot be explained in terms of physical concepts alone. DFTB+ performs well when compared with DFT, and lends itself to fast calculations for large systems.
\end{abstract}

\section{Introduction}

Carbon is the atom of life and of organic chemistry with its myriads of compounds. Even the elemental forms of carbon have intriguing properties: There are the extremely hard but metastable $s p^{3}$ bonded diamond, and a variety of $s p^{2}$ bonded forms, where one $p$ electron forms delocalized $\pi$ bonds. The latter materials are characterized by a low electronic density of states (DOS) at the Fermi level, or in the case of semiconducting carbon nanotubes (CNTs) by a small band gap of the order of $1 \mathrm{eV}[2,3]$. Therefore, their electronic properties are easily modified by doping, by adsorption, even by an external electric field. In particular, doping can convert graphene and CNTs, which in their pure form are not very reactive, to catalysts for a variety of reactions such as oxygen reduction or hydrogen evolution $[4,5]$.

In our group, we have been concerned with the interaction of CNTs with ions. Triggered by the observation of anomalously high capacitances of CNTs [6-8], and the

Wolfgang Schmickler

Wolfgang.Schmickler@uni-ulm.de

Fernanda Juarez

fernanda.juarez@uni-ulm.de

1 Institute of Theoretical Chemistry, Ulm University, D-89069, Ulm, Germany concept of a superionic state [9], we have investigated the insertion of ions into narrow tubes and their resulting effects such as the formation of image charges on the walls of the tubes, and the shift of the Fermi level, which can locally convert semiconducting into conducting tubes [10-12]. Recently, we discovered an unexpected effect: The virtual attraction between two lithium ions separated by the wall of a nanotube, which we explained by the shift in the Fermi level of the tube and the concomitant work required - the direct Coulomb interaction between the ions is negligible since it is screened by the tube [14]. Perhaps less surprisingly, we found an effective attraction between $\mathrm{a} \mathrm{Cl}^{-}$ion inside and $\mathrm{a} \mathrm{Li}^{+}$ion outside the tube, which we could explain by changes in the work function [1]. Thus, both effects could be explained in terms of physical concepts. In this work we investigate the interaction between two $\mathrm{Cl}^{-}$ions inside and outside a nanotube, where chemical effects dominate: Both the inserted ion inside and the adsorbed ion outside form a chemical bond with the nanotube. Nevertheless, in the investigated cases, the two ions attract each other, but the explanations involve chemical arguments in terms of bond formation.

The rest of this paper is organized as follows: First, we present the systems investigated and the methods of calculation employed. Then, we consider the bond between a single chloride ion inserted into a CNT or adsorbed outside. Next, we calculate the interaction between two ions situated inside and outside the tube, before we give 
a qualitative explanations in terms of bond formation and changes in the electronic densities of states.

\section{Systems and methods}

As in our previous work, we have chosen two model nanotubes: A semiconducting $(8,0) \mathrm{CNT}$ with radius $R=$ $3.2 \AA$, and a conducting $(5,5) \mathrm{CNT}$ with $R=3.5 \AA$. The former has a band gap centered about the Fermi level, while the latter has a small but finite DOS at the Fermi level. As mentioned in the "Introduction," we investigate the interaction of $\mathrm{Cl}^{-}$ions with these tubes.

The main part of the calculations has been performed with density functional theory including dispersion corrections using the SIESTA code. Cyclic boundary conditions have been employed. Since full DFT calculations are timeconsuming and allow only calculations for relatively short tubes, we have used this opportunity to test an attractive alternative: The DFT-based tight binding method as implemented in the program DFTB+. Since much of our understanding of graphene and CNTs rests on simple, tight binding arguments, we were optimistic that it would also give good results in our case. As we will show, our optimism was justified. All the technical details of our calculations are reported in the Appendix.

\section{Interaction of single $\mathrm{Cl}^{-}$ion with carbon nanotubes}

Here, we consider both the insertion of a $\mathrm{Cl}^{-}$ion into a CNT and the adsorption outside. In order not to duplicate the presentation, throughout this paper, we focus on the $(8,0) \mathrm{CNT}$, but give also the results for the $(5,5) \mathrm{CNT}$, which behaves very similarly. The insertion has been investigated before $[10,11]$, we briefly summarize the main points. Note that the insertion energies obtained here differ slightly from those previously published, where the dispersion interaction was not considered.
For both tubes, the stable position of the $\mathrm{Cl}^{-}$is at the center of the tube. When a $\mathrm{Cl}$ atom is placed inside a $\mathrm{CNT}$, an electron is transferred from the tube to form a $\mathrm{Cl}^{-}$ ion surrounded by a positive image charge on the wall of the tube (see Fig. 1 a). The center of the image charge is closer to the ions than the ring of carbon atoms. It can be characterized by the effective radius of the image charge, which is $R_{\mathrm{im}}=2.14 \AA$ for the $(8,0) \mathrm{CNT}$, and $R_{\mathrm{im}}=2.01$ $\AA$ for the $(5,5) \mathrm{CNT}[11,12]$. The difference between the physical and the image radius is larger for the $(5,5) \mathrm{CNT}$ $(1.35 \AA)$ than for the $(8,0) \mathrm{CNT}(1.04 \AA)$, because the former is a conductor even in the absence of the ion, and therefore screens somewhat better. Please note that some authors prefer the term " polarization charge" instead of "image charge"; we prefer the latter terminology in order to point out the similarity between the effective image radius for a tube and the effective position of the image plane at a metal surface [13].

For the adsorption of the ion outside the CNTs with DFT, we found a stable position on top of a carbon atom, and a metastable position above the center of a carbon ring; DFTB+ only gave us the stable site. The charge distribution in Fig. 1 shows that the metastable state, which is at a larger distance, has the character of an ion, while at the stable position the charge of the $\mathrm{Cl}^{-}$is smaller, and there is a small electronic excess charge at the center of the bond with the tube, which indicates that this bond has a substantial covalent contribution.

The insertion and adsorption energies are given in Table 1; the bonding distances in Table 2 . The energies $E_{1}$ of interaction (insertion or adsorption) between one ion and the tube have been calculated as follows:

$E_{1}=E_{\mathrm{CNT}+\mathrm{Cl}}-E_{\mathrm{CNT}}-E_{\mathrm{Cl}}$

where $E_{\mathrm{CNT}+\mathrm{Cl}}, E_{\mathrm{CNT}}$, and $E_{\mathrm{Cl}}$ are the energies of the ion and the nanotube, the pure tube, and the $\mathrm{Cl}$ atom in the vacuum, respectively. Clearly, the insertion of a $\mathrm{Cl}^{-}$ion is a more favorable process than its adsorption outside of the nanotube. This is not surprising since the inserted ion is surrounded by carbon atoms on all sides. The energy

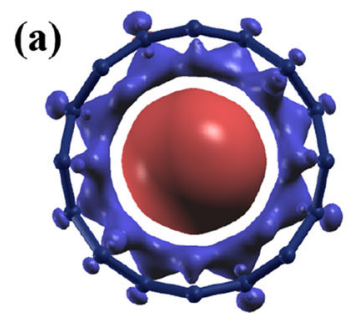

(b)

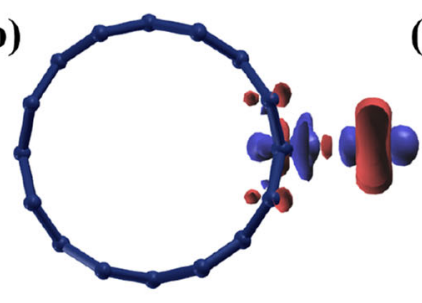

(c)

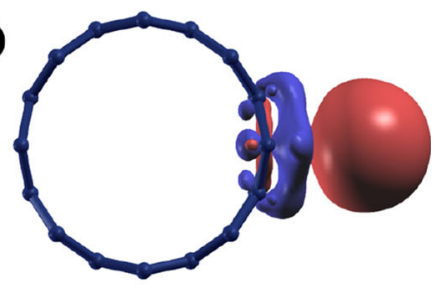

Fig. 1 Electronic density differences $\Delta \rho$ of $\mathrm{Cl}^{-}$on an $(8,0) \mathrm{CNT}$. The adsorption sites are (a) inside, (b) top site (outside), and (c) center of ring (outside). The density difference was calculated as $\Delta \rho=$ $\rho_{\mathrm{CNT}+\mathrm{Cl}}-\rho_{\mathrm{CNT}}-\rho_{\mathrm{Cl}}$. Carbon and chloride atoms are plotted as blue and green spheres, respectively. The isovalues correspond to \pm 0.001 $\mathrm{e} \AA^{-3}$, where a negative (or positive) excess charge is shown in red (or blue) 
Table 1 Interaction energies $E_{1}$ of chloride ions with the CNTs calculated with SIESTA and DFTB+ codes

\begin{tabular}{lllll}
\hline Position & $(8,0) \mathrm{CNT}$ & $(8,0) \mathrm{CNT}+$ & $(5,5) \mathrm{CNT}$ & $(5,5) \mathrm{CNT}$ \\
& SIESTA & DFTB & SIESTA & DFTB+ \\
\hline Inside-middle & -1.65 & -1.30 & -1.69 & -1.77 \\
Outside-top & -1.04 & -1.15 & -0.95 & -1.32 \\
Outside-c-ring & -0.87 & & -0.83 & \\
\hline
\end{tabular}

c-Ring denotes a site above the center of a carbon ring. All the values are in $\mathrm{eV}$

difference between the two sites outside the tube is similar for both CNTs (ca. $0.15 \mathrm{eV}$ ). The top position, where the ion is closer to the carbon atoms, is the preferred site.

The results obtained with DFTB+ code are in good agreement with the SIESTA calculations, and both of them show the same trends. In general, the tight-binding code seems to overestimate the values with respect to DFT, with the exception of the insertion into the $(8,0)$ nanotube. We note that different flavors of DFT based on slightly different functionals also tend to give results that differ by an order of $\pm 0.1 \mathrm{eV}$.

The bonding and the charge distribution can be studied in greater detail by investigating the densities of states (DOS). The situation is very similar for the two tubes, so we present the results for the $(8,0)$ CNT. Figure 2 shows the DOS corresponding to the systems of Fig. 1 (a)-(c), and projected onto the contributions of the $\mathrm{Cl}$ and $\mathrm{C}$ atoms. The DOS of the pure nanotube is shown in the background of each picture (in gray), and it has been shifted in energy so that the main features coincide-in the pristine tube, the Fermi level is at the center of the gap.

When the ion is adsorbed inside the tube, the DOS of the carbon atoms is shifted by about $0.3 \mathrm{eV}$ toward positive values, so that the top of the valence band just rises above the Fermi level. The $\mathrm{Cl}^{-}$ion gives rise to two narrow peaks directly below the Fermi level, which correspond to different spin orientations, and which indicate a negative excess charge and an absence of covalent bonding. The positive counter charge is distributed on the carbon atoms, so that the charge on each atom is small. Since the tubes investigated with Siesta are comparatively short, we have calculated the distribution of the counter charge with DFTB+, for which tubes with a length of $40 \AA$ pose no problem. The details can be seen in Fig. 3. The positive charge on the carbon atoms is centered at the position of the $\mathrm{Cl}^{-}$ions, and falls off over a distance of about $10 \AA$. On the $(5,5) \mathrm{CNT}$, the decay of the excess charge is monotonous; in contrast, the $(8,0) \mathrm{CNT}$ shows small, but distinct Friedel oscillations [15] because of its low electronic density near the Fermi level.
Table 2 Distances between the chloride ions and the wall of the CNTs calculated with SIESTA and DFTB+ codes

\begin{tabular}{lllll}
\hline Position & $\begin{array}{l}(8,0) \mathrm{CNT} \\
\text { SIESTA }\end{array}$ & $\begin{array}{l}(8,0) \mathrm{CNT} \\
\text { DFTB+ }\end{array}$ & $\begin{array}{l}(5,5) \mathrm{CNT} \\
\text { SIESTA }\end{array}$ & $\begin{array}{l}(5,5) \mathrm{CNT} \\
\text { DFTB+ }\end{array}$ \\
\hline Inside-middle & 3.24 & 3.20 & 3.55 & 3.51 \\
Outside-top & 2.11 & 2.36 & 2.22 & 2.40 \\
Outside-c-ring & 2.67 & & 3.00 & \\
\hline
\end{tabular}

c-Ring denotes a site above the center of a carbon ring. All the values are in $\AA$

When the ion is adsorbed above the center of a ring outside (Fig. 2c), the DOS resembles that of the case where the ion is inside. However, the splitting between the two spin states is larger, and the DOS of the down spin extends a little above the Fermi level, indicating that the charge is smaller. Also, the lower of the two spin states shows some overlap with a carbon peak, indicating a small covalent contribution to the bond. Otherwise, there is little change in the carbon DOS, while for the inserted ion there are some new oscillations in the range between -1.5 and $-2 \mathrm{eV}$. Thus, the DOS indicates that for the inserted ion and for the metastable adsorption (c) the bond with the tube is mainly ionic.

The DOS of the chloride ion adsorbed on the top position is rather different from that of the other two systems. The states are less localized and deeper in energy. The electronic structure of the tube has also been modified, especially near the Fermi level where new states have been created. The shift of the DOS of the pure nanotube is smaller in this case (only $0.1 \mathrm{eV}$ ), and so is the charge transfer between the $\mathrm{C}$ and the $\mathrm{Cl}$ atoms. This is another indication that the bond is less ionic in this position.

\section{Energetics of the interaction of a pair of $\mathrm{Cl}^{-}$ ions across CNTs}

In this section, we study the interaction of a $\mathrm{Cl}^{-}$ion inside with another anion outside of the nanotube. The inside ion was fixed at its optimum position at the center of the tube whereas the position of the ion outside was varied systematically. For the $(8,0) \mathrm{CNT}$, the optimized path is shown in Fig. 4 (b). The ion inside is placed at $x=0$; the closest position outside is a bridge site in front of the chloride. The optimal path follows the shape of the carbon hexagons, going from top to top sites through bridge positions. Some of the systems, where the $\mathrm{Cl}^{-}$ion outside is on top, have been labelled from $\mathrm{A}$ to $\mathrm{E}$, from closer to farther to the ion inside, respectively. Because of the symmetry of the nanotube, paths going upwards or downwards are equivalent. 

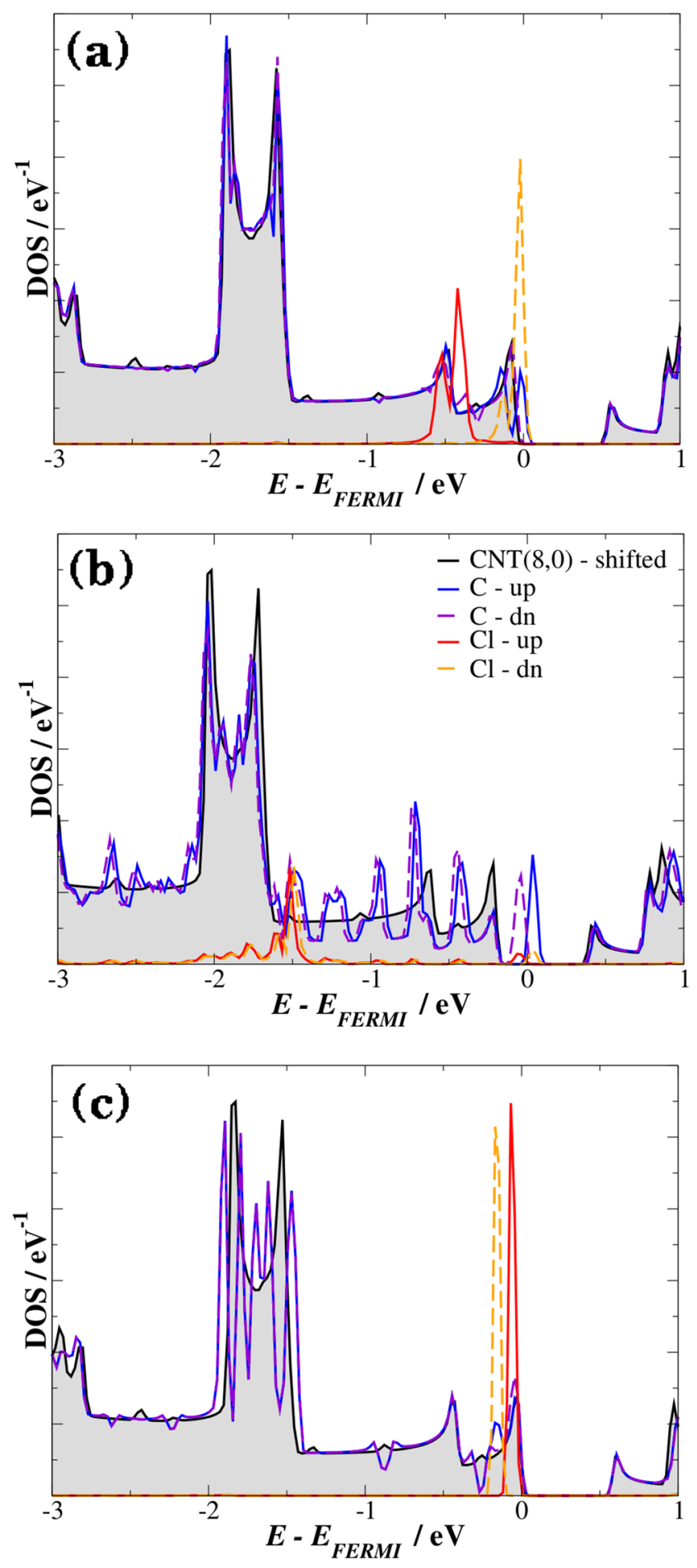

Fig. 2 DOS of $\mathrm{Cl}^{-}$on the (8,0)CNT in different sites: (a) inside, (b) on top (outside), and (c) above the center of a carbon ring (outside). The total DOS of the pure $(8,0) \mathrm{CNT}$ is displayed in the background of the three pictures

The energies $E_{2}$ of adsorption of the second ion on the nanotube with one ion inside were calculated as follows:

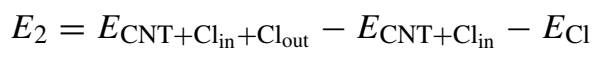

where $E_{\mathrm{CNT}+\mathrm{Cl}_{\text {in }}+\mathrm{Cl}_{\text {out }}}$ is the energy of the system with two ions. Using this equation, the energies of adsorption were calculated as a function of the axial coordinate of the ion outside of the tube (red line in Fig. 4 (a)). For comparison, the interaction energies $E_{1}$ of one ion outside of the empty nanotube were calculated using the same positions as in Fig. 4 (b).

In both curves, the maximum energy values stand for $\mathrm{Cl}^{-}$adsorbed on the bridge position, and the minima to adsorption on top. The lower energies for the adsorption of the ion outside in the presence of the ion inside show that there is a virtual attraction between the two chloride ions. This effect increases when the two ions get closer, obtaining a maximum value of $0.27 \mathrm{eV}$ in position $\mathrm{A}$. The difference in energy $E_{2}$ between sites $\mathrm{E}$ and $\mathrm{A}$ is another effect of the order of $0.07 \mathrm{eV}$. The attractive interaction is also more noticeable on the top than on the bridge sites.

Repeating the same procedure as before, we have calculated the interaction energies of two ions on the $(5,5) \mathrm{CNT}$. The ideal path and the corresponding energies are shown in Fig. 5. Here, again, the $\mathrm{Cl}^{-}$ion outside follows the shape of the carbon hexagons going from one minima in a top position to another through the bridge sites (black curve). Similar to the case of the semiconducting tube, a virtual attraction between the chloride ions is observed, which is of the same order of magnitude when they are closer (in position A).

In the $(8,0) \mathrm{CNT}$, the same attractive effect was observed using the DFTB+ code (see Table 3). However, the attraction was significantly smaller on the conducting $(5,5)$ tube, but the absolute difference is small. Using both methods, we found that the systems are more stable when the ions are closer.

\section{Electronic structure of a pair of $\mathrm{Cl}^{-}$ions on CNTs}

In order to understand the attractive effect between the chloride ions, we have analyzed the changes in the electronic structure by using the DOS profiles; again, we focus on the $(8,0) \mathrm{CNT}$. Figure 6 shows the projected DOS of the system with inside and outside ions adsorbed on the $(8,0)$ CNT. In general, the profile of the carbon atoms is very similar to the one shown in Fig. 2 (b), where there is only one ion adsorbed outside of the tube (on top position). As in the previous case, there are also a few new states created near the Fermi level, and the DOS of the tube has been slightly shifted to higher energies due to the presence of the ion inside. The states corresponding to the $\mathrm{Cl}^{-}$ion inside are very localized and placed near the Fermi level; they are hardly affected by the outside ion. Only the states of the ion outside have been significantly modified due to the presence 
Cl-in $(8,0)$ CNT

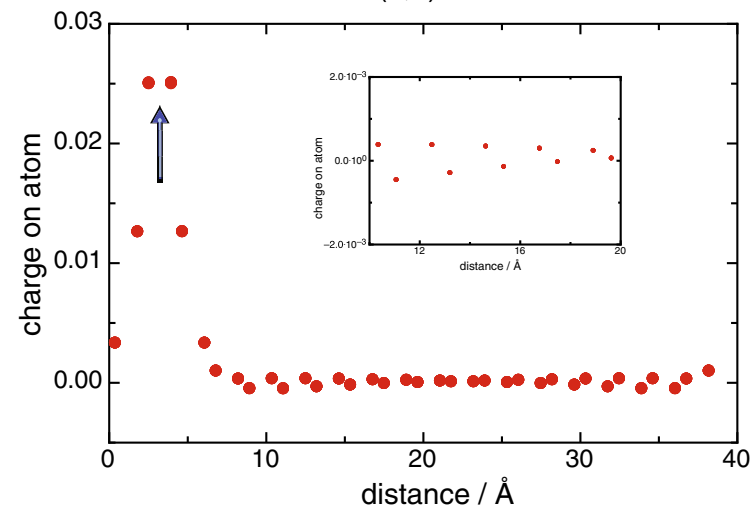

Cl-in $(5,5) \mathrm{CNT}$

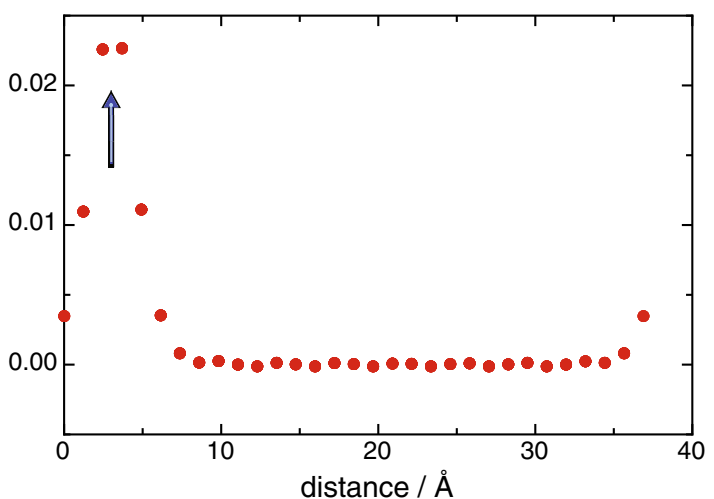

Fig. 3 Distribution of the charge on the carbon atoms along the tube. For reasons of symmetry, each point represents 8 atoms for the $(8,0) C N T$, and 10 atoms for the $(5,5) \mathrm{CNT}$. The position of the $\mathrm{Cl}^{-}$inside is indicated by the blue arrow. Note the periodic boundary conditions. Results obtained with DFTB+

Fig. 4 (a) Interaction energies $E_{1}$ and $E_{2}$ between $\mathrm{Cl}^{-}$ions and $(8,0) \mathrm{CNT}$. All the energies are in eV. (b) Optimal geometries of the systems with 2 $\mathrm{Cl}^{-}$ions. The inside ion is shown in red and the optimal geometries of the ion outside (in green) have been superimposed. Each system has only 2 ions
Fig. 5 (a) Interaction energies $E_{1}$ and $E_{2}$ between $\mathrm{Cl}^{-}$ions and $(5,5) \mathrm{CNT}$. All the energies are in eV. (b) Optimal geometries of the systems with 2 $\mathrm{Cl}^{-}$ions. The inside ion is shown in red and the optimal geometries of the ion outside (in green) have been superimposed. Each system has only two ions
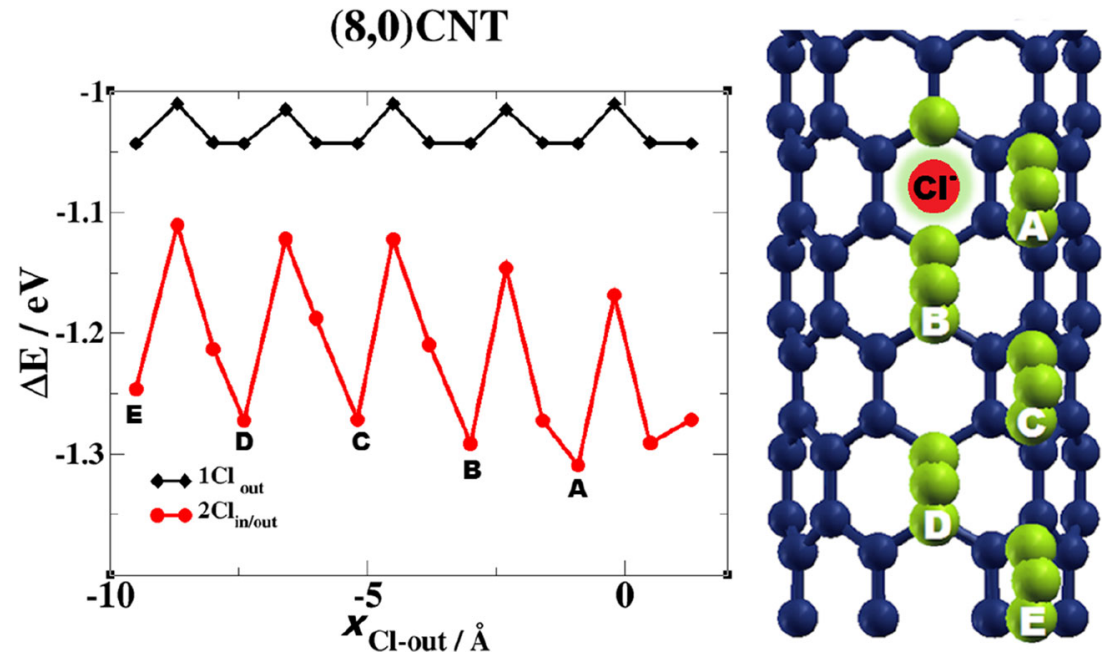

(a)

$(5,5) \mathrm{CNT}$

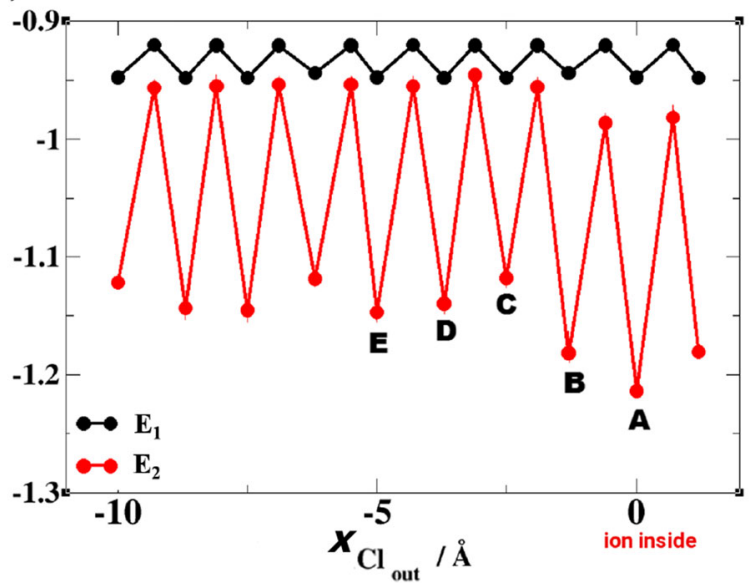

(b)

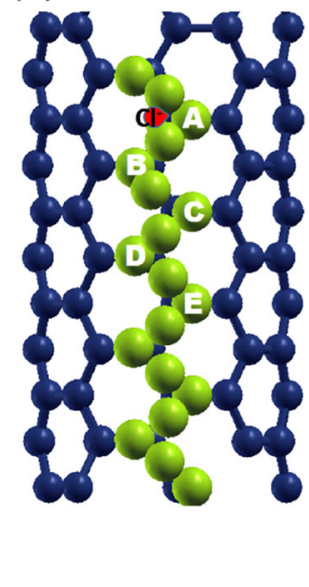


Table 3 Interaction energies of ions across the carbon nanotubes, calculated with DFTB+ and SIESTA code

\begin{tabular}{|c|c|c|c|c|}
\hline \multirow[t]{2}{*}{$E_{2}-E_{1}$} & \multicolumn{2}{|c|}{$(8,0) \mathrm{CNT}$} & \multicolumn{2}{|l|}{$(5,5) \mathrm{CNT}$} \\
\hline & SIESTA & DFTB+ & SIESTA & DFTB+ \\
\hline Position A & -0.27 & -0.32 & -0.26 & -0.10 \\
\hline Position E & -0.20 & -0.15 & -0.17 & -0.01 \\
\hline
\end{tabular}

All the values are in $\mathrm{eV}$

of the inside ion. Comparing Figs. 2 (b) and 6, the maximum is moved from -1.5 to $-2.1 \mathrm{eV}$, where it shows a significant overlap with a new carbon peak, which indicates bonding. A similar change occurs in the case of the $(5,5)$ nanotube, where the maximum of the p-orbital moves from -1.1 to $-1.9 \mathrm{eV}$. These shifts in the DOS of the outside ion towards lower energies entail a lowering of the energy, and appear like a virtual attraction.

In a previous work, we have explained the apparent attraction between lithium ions as the result of the modification of the electronic structure of the nanotube. In that case, the transfer of charge from the lithium to the tube transforms the semiconducting CNT into a conducting one, facilitating the adsorption of the second cation. The effect was considerably smaller in a conducting tube. In contrast to that case, the adsorption of the anions modifies the valence band of the nanotube producing a very similar effect on conducting and semiconducting nanotubes.

To explain the decrease in energy when the two anions get closer, we have plotted the partial DOS of the $\mathrm{Cl}^{-}$ions inside and outside the $(8,0) \mathrm{CNT}$ for the cases where the ions are farther and closer to each other (E and A in Fig. 4). The pictures are shown in Fig. 7. When the ions are closer,

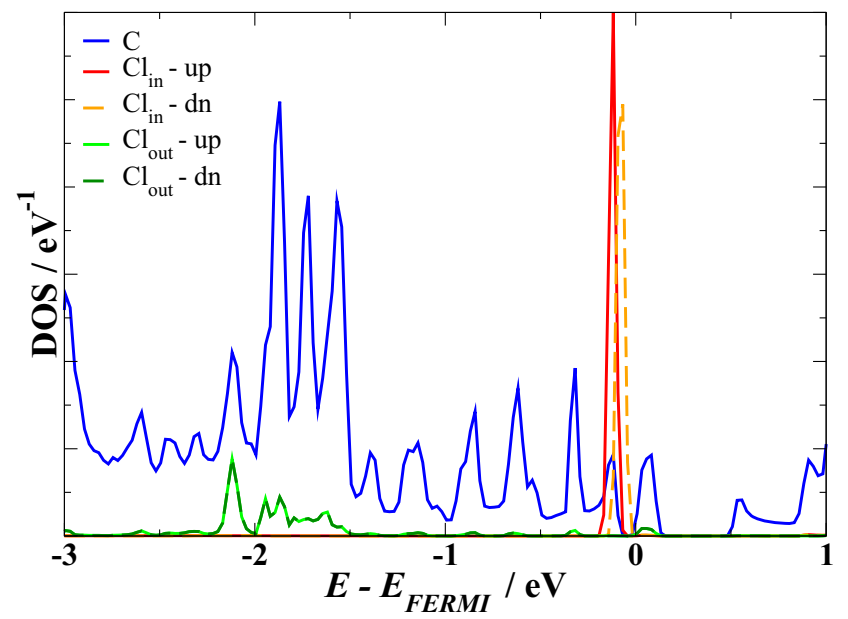

Fig. 6 DOS of two $\mathrm{Cl}^{-}$ions on the $(8,0) \mathrm{CNT}$. The system corresponds to position $\mathrm{E}$ in Fig. 4. The arrow indicates the overlap of a $\mathrm{C}$ and $\mathrm{a} \mathrm{Cl}$ peak, which indicates bonding
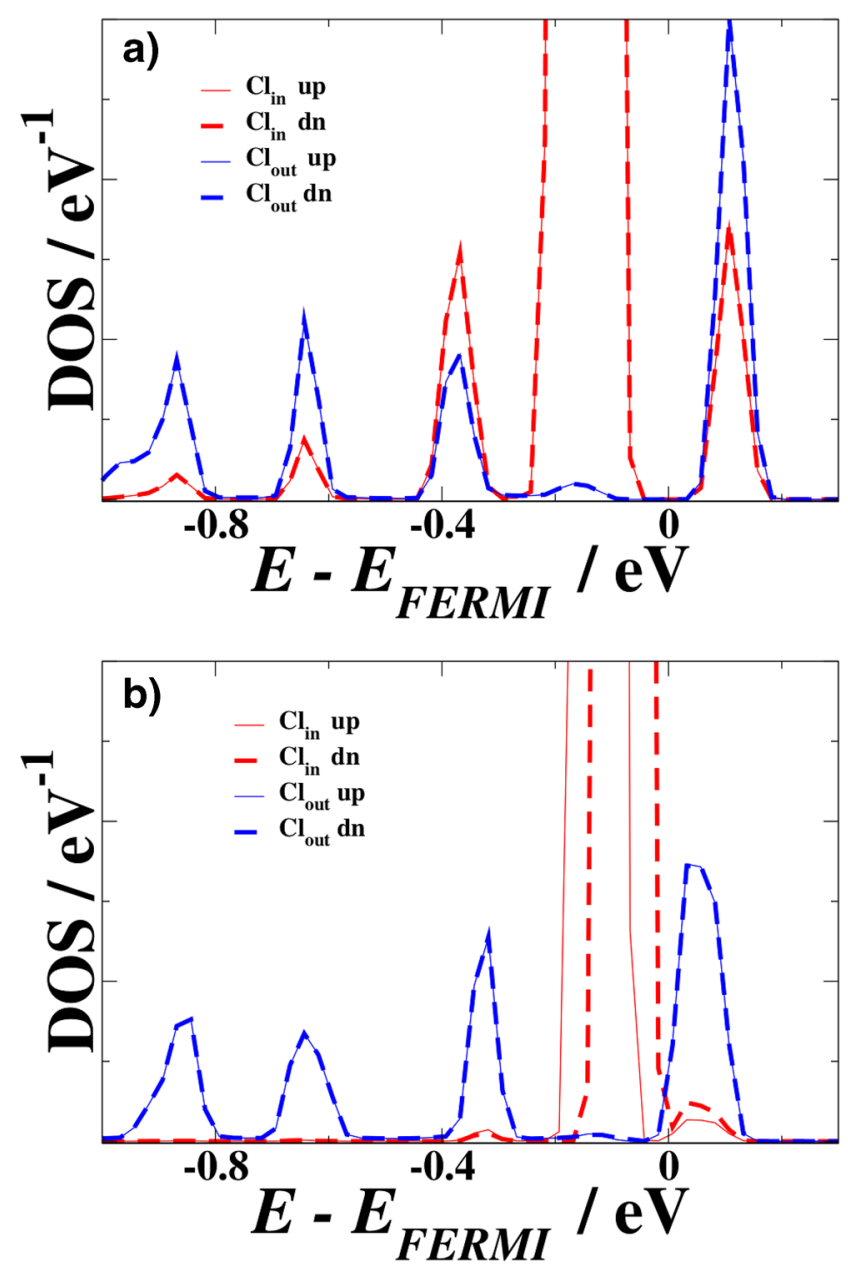

Fig. 7 Partial DOS of two $\mathrm{Cl}^{-}$ions on the $(8,0) \mathrm{CNT}$. The system corresponds to (a) position A, and (b) position E in Fig. 4

there is no splitting of the spins and, most importantly, several peaks of the two ions coincide (see the arrows). This indicates a direct interaction between them that is not present when the ions are far apart. We surmise that this is the reason why the attraction is strongest when they are close.

\section{Conclusions}

This article concludes our investigations of the interaction of simple ions across carbon nanotubes. Like in the case of two $\mathrm{Li}^{+}$ions, we observed an effective attraction between the two chloride ions: The energy of adsorption of an outside ion was lower (more favorable) in the presence of an inside ion. In all three cases, $\mathrm{Li}^{+}-\mathrm{Li}^{+}, \mathrm{Cl}^{-}-\mathrm{Li}^{+}$, and $\mathrm{Cl}^{-}-$ $\mathrm{Cl}^{-}$, the attraction was mediated by the carbon nanotubes that separate the pairs. While the first two cases could be explained in terms of physical effects, the present case is the most difficult because the interaction of the ions with the 
nanotubes is chemical; in particular that of the outside ion has a strong covalent component. In essence, the presence of the inside ion shifts the DOS of the outside ion towards lower energies, and thus lowers the energy of the adsorbate. When the two $\mathrm{Cl}^{-}$ions are close, there is even a direct covalent interaction, which manifests itself in the overlap of peaks in the DOS. Therefore, the attractive effect is larger when the ions are close. This contrasts with the case of $\mathrm{Li}^{+}$ $-\mathrm{Li}^{+}$, where the virtual attraction was weaker when the ions are close because of a weak residual penetration of the direct Coulomb interaction.

An important point is that the direct interaction between the ions is screened by the nanotube, no matter if it is a conducting or a semiconducting tube. This seems to contradict theories for the screening of charges in CNTs, which state that semiconducting tubes cannot screen an external charge [18]. However, these theories are based on linear response. In contrast, in our calculations, the response was so strong that the semiconducting tube became locally conductive.

We have used the opportunity to test the DFT-based tight binding method DFTB+ as a fast alternative to normal DFT. On the whole, the results are very encouraging: the method lends itself to performing fast calculations for larger systems than pure DFT can handle.

Acknowledgments W.S. and E.S. thank CONICET for continued support. F.D.-F. would like to thank Consejo Nacional de Ciencia y Tecnología (Mexico) for a scholarship. We thank Dr. Bálint Aradi from Bremen Center for Computational Materials Science, University of Bremen, for useful advice on DFTB+.

Funding information Open Access funding provided by Projekt DEAL. We received financial support by the Deutsche Forschungsgemeinschaft (Schm344/48-1,48-2). The authors received support by the state of Baden-Württemberg through bwHPC and the German Research Foundation (DFG) through grant no. INST 40/467-1 FUGG (JUSTUS cluster).

Open Access This article is licensed under a Creative Commons Attribution 4.0 International License, which permits use, sharing, adaptation, distribution and reproduction in any medium or format, as long as you give appropriate credit to the original author(s) and the source, provide a link to the Creative Commons licence, and indicate if changes were made. The images or other third party material in this article are included in the article's Creative Commons licence, unless indicated otherwise in a credit line to the material. If material is not included in the article's Creative Commons licence and your intended use is not permitted by statutory regulation or exceeds the permitted use, you will need to obtain permission directly from the copyright holder. To view a copy of this licence, visit http:// creativecommonshorg/licenses/by/4.0/.

\section{Appendix 1}

\section{Technical details of the simulations performed with SIESTA}

The electron-ion interactions were accounted through ultrasoft pseudopotentials [16], while the valence electrons were treated within the van der Waals approximation ( $\mathrm{vdW})$ in the version of non-local vdW correlation functional DSRLL [17]. A grid spacing was set, corresponding to an effective energy cutoff of 300 Ry. The Brillouin zone integration was performed by creating a grid in the axial direction of the tube with a separation of $5 \AA$ per k-point and starting from the Gamma point. In order to calculate the density of states, a finer grid of 70 k-points was used. The infinite nanotubes were described using periodic boundary conditions and cells with size: $(19.93 \times 25.0 \times 25.0) \AA^{3}$ and $(21.55 \times 25.0 \times 25.0) \AA^{3}$ for the $(5,5)$ and $(8,0) \mathrm{CNTs}$, respectively. The optimized diameters were respectively 6.99 and $6.47 \AA$.

\section{Technical details of the simulations performed with DFTB+}

The total energy was calculated using the third-order density functional based tight binding method (called DFTB3) [19]. The full 3rd-order corrections to the SCC-Hamiltonian were done using Hubbard derivatives: -0.1492 and -0.0697 for $\mathrm{C}$ and $\mathrm{Cl}$, respectively [20]. The interactions between pairs of atoms were calculated using the latest version of the $3 \mathrm{OB}$ set of Slater-Koster files [20]. The van der Waals energy terms were included using the D3-Grimme's dispersion correction with the Becke-Johnson damping scheme, parametrized for the selected Slater-Koster files [21].

In all the calculations, we used periodic boundary and the reciprocal space was sampled using a $20 \mathrm{k}$-points grid and the Monkhorst-Pack method. The size of the cells were: $(19.80 \times 50.0 \times 50.0)$ and $(21.40 \times 50.0 \times 50.0) \AA^{3}$ for the $(5,5)$ and $(8,0) \mathrm{CNTs}$, respectively. The optimized diameters were respectively 6.9 and $6.4 \AA$.

\section{References}

1. Juarez F, Dominguez-Flores F, Quaino P, Santos E, Schmickler W (2020) Interactions between ions across carbon nanotubes. Phys Chem Chem Phys 22:10603

2. Bachilo SM, Strano MS, Kittrell C, Hauge RH, Smalley RE, Weisman RB (2002) Structure-assigned optical spectra of singlewalled carbon nanotubes. Science 298:2361 
3. Weisman RB, Bachilo SM (2003) Dependence of optical transition energies on structure for single-walled carbon nanotubes in aqueous suspension: an empirical Kataura plot. Nano Lett 3:1235

4. Muhammad AY, Yang $\mathrm{H}$ Advanced nanomaterials for pollutant sensing and environmental catalysis., 'Chapter 10 - nanocarbon-based hybrids as electrocatalysts for hydrogen and oxygen evolution from water splitting. https://doi.org/10.1016/B978-0-12-814796-2.00010-1

5. Qu K, Zheng Y, Zhang X, Davey K, Dai S, Qiao SZ (2017) Promotion of electrocatalytic hydrogen evolution reaction on nitrogen-doped carbon nanosheets with secondary heteroatoms. ACS Nano 11:7293-7300

6. Chmiola J, Yushin G, Gogotsi Y, Portet C, Simon P, Taberna P (2006) Anomalous increase in carbon capacitance at pore sizes less than 1 nanometer. Science 313:1760

7. Largeot C, Portet C, Chmiola J, Taberna P-L, Gogotsi Y, Simon P (2730) Relation between the ion size and pore size for an electric double-layer capacitor. J Am Chem Soc 2008:130

8. Xing L, Vatamanu J, Borodin O, Bedrov D (2013) On the atomistic nature of capacitance enhancement generated by ionic liquid electrolyte confined in subnanometer pores. J Phys Chem Lett 4:132

9. Kondrat S, Kornyshev A (2010) Superionic state in double-layer capacitors with nanoporous electrodes. J Phys Condens Matt 23(2):022201

10. Goduljan A, Juarez F, Mohammadzadeh L, Quaino P, Santos E, Schmickler W (2014) Screening of ions in carbon and gold nanotubes a theoretical study. Electrochem Comm 45:48

11. Mohammadzadeh L, Goduljan A, Juarez F, Quaino P, Santos E, Schmickler W (2015) Nanotubes for charge storage towards an atomistic model. Electrochim Acta 162:11
12. Mohammadzadeh L, Goduljan A, Juarez F, Quaino P, Santos E, Schmickler W (2016) On the energetics of ions in carbon and gold nanotubes. Chem Phys Chem 17:78

13. Lang ND, Kohn W (1971) Theory of metal surfaces: work function. Phys Rev B 3:1215. Theory of metal surfaces: induced surface charge and image potential, 7:1430E (1973)

14. Juarez F, Dominguez-Flores F, Goduljan A, Mohammedzadeh L, Santos E, Schmickler W (2018) Defying Coulomb's law: a lattice-induced attraction between lithium ions. Carbon 139:808

15. Friedel J (1954) Electronic structure of primary solid solutions in metals. Adv Phys 3:346

16. Vanderbilt D (1990) Soft self-consistent pseudopotentials in a generalized eigenvalue formalism. Phys Rev B 41:7892

17. Dion M, Rydberg H, Schröder E, Langreth DC, lundqvist BI (2004) Van der Waals density functional for general geometry. Phys Rev Lett 246401:92

18. Lin MF, Chu DS (1997) Impurity screening in carbon nanotubes. Phys Rev B 56:4996

19. Gaus M, Cui Q, Elstner M (2011) DFTB3: extension of the selfconsistent-charge density-functional tight-binding method (SCCDFTB). J Chem Theor Comput 7:931-948

20. Kubillus M, Kubar T, Gaus M, Rezac J, Elstner M (2015) Parameterization of the DFTB3 method for $\mathrm{Br}, \mathrm{Ca}, \mathrm{Cl}, \mathrm{F}, \mathrm{I}, \mathrm{K}$, and $\mathrm{Na}$ in organic and biological systems. J Chem Theor Comput 11:332-342

21. Grimme S, Ehrlich S, Goerigk L (2011) Effect of the damping function in dispersion corrected density functional theory. $\mathrm{J}$ Comput Chem 32:1456

Publisher's note Springer Nature remains neutral with regard to jurisdictional claims in published maps and institutional affiliations. 